KAIST-CHEP-94/08

\title{
Derivation of the Classical Lagrangian for the Relativistic Spinning Particle
}

\author{
Jin-Ho Cho f and Jae-Kwan Kim \\ Department of Physics \\ Korea Advanced Institute of Science and Technology \\ 373-1 Yusung-ku, Taejon, 305-701, Korea
}

\begin{abstract}
The 'classical' model for a massive spinning particle, which was recently proposed, is derived from the isotropic rotator model. Through this derivation, we note that the spin can be understood as the relativistic extension of the isotropic rotator. Furthermore, the variables $t_{\mu}$ corresponding to the $\psi^{*}$ of the 'pseudo-classical' model, are necessary for the covariant formulation. The dynamical term for these extra variables is naturally obtained and the meaning of the constraint term $p^{\sigma} \Lambda_{\sigma \nu}+m t_{\nu}=0$, which was recently shown to give 'quasi-supersymmetry', is clarified.
\end{abstract}

*jhcho@chiak.kaist.ac.kr 
Since the discovery of the spin effect by Stern-Gerlach experiment in 1922, several attempts have been made to understand the 'spin' concept. Motivating the departure from the 'classical' theory, 'spin' has long been understood as the 'quantum' effect.

However, since the explanation of spin via 'Zitterbewegung' by Schrödinger [1], we still search for some classical means to understand the spin [2]. By the introduction of supersymmetry [3], we came to know that spin is somewhat 'relativistic' effect rather than 'quantum' concept. Those 'pseudo-classical' formulations use Grassmann variables to describe the spin degree of freedom. We also can use cnumber spinor for that [4]. Besides this historical aspect, the relativistic spinning particle has been investigated extensively due to its rich structure [5].

In other direction, there have been some studies on the geometrical construction of spin using group variables [6]. Furthermore, by the modification of this 'classical' model, some analogous features (constraint structure and supersymmetry) with the pseudo-classical model were found [7]. Moreover, Polyakov's spin factor [8] can be obtained via BRST invariant path integral [9].

In this letter, we clarify those concepts concerning the geometrical meaning of the spin, the constraints and the variables $t^{a}$ corresponding to $\psi^{*}$ of the pseudo-classical model, through the derivation of the model recently proposed in [7]. We start from the isotropic rotator model and proceed to replace the variables, introduced to specify the orientation of the rotator, with $S O(3)$ group variables. Further we give local $S O(3)$ symmetry to describe the variables with respect to the general rotating frame. Finally, we transfer to the relativistic case.

To describe the non-relativistic free spinless particle, we use the usual position variables $x^{i}$. The Lagrangian is then given by

$$
\mathcal{L}_{\text {posit }}=\frac{1}{2} m \dot{x}^{2} \text {. }
$$

For the non-relativistic isotropic rotator, in addition to the above variables specifying the position of the center of mass, we introduce other variables: the coordinates $r^{i}(t)$ for a point of the rotator body with respect to the non-rotating frame', to take into account the orientation of the rotator;

$$
\begin{aligned}
r(t) & =O(t) R_{0} \\
\dot{r}(t) & =\dot{O}(t) O^{-1}(t) r(t) \\
& \equiv \varpi(t) r(t)=w(t) \times r(t),
\end{aligned}
$$

\footnotetext{
${ }^{1}$ We should discriminate the non-rotating frame, which has its origin at the center of mass and moves with the rotator, from the observer-rest inertial frame where the observer resides.
} 
where $R_{0}$ denote the coordinates for that point of the rotator body with respect to the body-fixed frame, thus is time independent? and $w(t)$ is the angular velocity of the rotator with respect to the non-rotating frame. We can also define the corresponding quantities with respect to the body-fixed frame;

$$
\begin{aligned}
\dot{r}(t) & =O(t) O^{-1}(t) \dot{O}(t) R_{0} \\
& \equiv O(t) \Omega_{0}(t) R_{0}=O(t)\left(W_{0}(t) \times R_{0}\right) \\
& \equiv O(t) V_{0}(t)
\end{aligned}
$$

The time dependency of the coordinates $r(t)$ is entirely due to the rotation $O(t) \in$ $S O(3)$ of the body. Therefore, we can use $O(t)$ as the orientation coordinates. In (2) 3) the following isomorphism between $\left\{\mathbb{R}^{3}, \times\right\}$ and $\{s o(3),[]$,$\} is to be noted;$

$$
\varpi(t)=w^{i}(t) L_{i}, \Omega_{0}(t)=\left(W_{0}\right)^{i}(t) L_{i},
$$

where the basis $L_{i}$ for $s o(3)$ is adjointly represented as $\left(L_{i}\right)_{j k}=-\epsilon_{i j k}$.

The Lagrangian for this orientation part is given by

$$
\begin{aligned}
\mathcal{L}_{\text {orient }} & =\frac{1}{2} \int \rho(r) \dot{r}^{2} d r=-\frac{1}{2} \iota(t) w(t)^{2} \\
& =-\frac{1}{2} I_{0} W_{0}^{2} \\
& =-\frac{1}{2}<\Omega_{0}(t), I_{0} \Omega_{0}(t)> \\
& =-\frac{1}{2}<O^{-1}(t) \dot{O}(t), I_{0} O^{-1}(t) \dot{O}(t)>,
\end{aligned}
$$

where $\rho(r)$ is the mass density of the rotator and $I_{0}=O^{-1}(t) \iota(t) O(t)$ is the moment of inertia tensor and $<,>=-\frac{1}{2} \operatorname{Tr}($,$) is the canonical Killing form?. Finally the$ Lagrangian for the free isotropic rotator becomes

$$
\mathcal{L}_{\text {rotator }}=\frac{1}{2} m \dot{x}^{2}-\frac{1}{2}<\tilde{S}_{0}(t), O^{-1}(t) \dot{O}(t)>,
$$

where $\tilde{S}_{0}(t) \equiv I_{0} O^{-1}(t) \dot{O}(t)$ is the angular momentum seen from the body-fixed frame. This system is very similar to the one in [6], where they construct the Lagrangian through topological argument. Furthermore that dynamical term is nothing but the canonical action that gives the symplectic structure on the group manifold [1].

\footnotetext{
${ }^{2}$ In the general rotating frame, $\dot{r}(t)=\dot{O}(t) R(t)+O(t) \dot{R}(t)=O(t)\left(\dot{R}(t)+O^{-1}(t) \dot{O}(t) R(t)\right) \equiv$ $O(t) D_{t} R(t)$, where $D_{t}=O^{-1} \frac{d}{d t} O=\frac{d}{d t}+O^{-1} \dot{O}(t)$ is the covariant derivative [10].

${ }^{3} I_{0}$ is symmetric with respect to this Killing form due to the trace property and thus can be diagonalizable. For the isotropic rotator, it is proportional to the identity.
} 
The system has the apparent global $S O(3)$ symmetry concerning the rotation of the observer-rest inertial frame.

$$
x(t) \rightarrow G x(t), r(t) \rightarrow G r(t), O(t) \rightarrow G O(t),
$$

where $G \in S O(3)$ and we note that $R_{0}$ is invariant under this global rotation. Together with this global symmetry we also expect a local $S O(3)$ symmetry. For $L(t) \in S O(3)$,

$$
\begin{aligned}
r(t) & =O(t) L^{-1}(t) L(t) R_{0} \\
& =O^{\prime}(t) R(t) \\
O(t) & \rightarrow O(t) L^{-1} \\
R_{0} & \rightarrow L(t) R_{0}
\end{aligned}
$$

This symmetry means the covariance of the system in the general rotating frame (a frame with its origin at the center of mass and generally rotating with respect to the body-fixed frame). We consider this symmetry to be internal in the sense that $x(t)$ and $r(t)$ are invariant. However $\Omega_{0}(t)=O^{-1}(t) \dot{O}(t)$ does not transform covariantly under this local internal symmetry;

$$
\Omega_{0}(t) \rightarrow L(t) \Omega_{0}(t) L^{-1}(t)+L(t) \dot{L}^{-1}(t)
$$

Because of the second affine term, the Lagrangian (6) is not invariant under the symmetry. To restore that local symmetry we replace the body fixed frame with the general rotating frame.

$$
\begin{aligned}
R_{0} & \Rightarrow \\
V_{0}(t) & \Rightarrow(t)=g(t) R_{0} \\
& D_{t} R(t)=\dot{R}(t)+O^{-1} \dot{O}(t) R(t) \\
& =\left(\dot{g}(t) g^{-1}(t)+\Omega(t)\right) R(t) \equiv(\mathcal{W}(t)+\Omega(t)) R(t) .
\end{aligned}
$$

Therefore $\Omega(t)$ is indeed the connection for this local symmetry and $\mathcal{W}(t)$ is the angular velocity of the rotator with respect to the general rotating frame.

We are now ready to covariantize the Lagrangian for the orientation part;

$$
\begin{aligned}
\mathcal{L}_{\text {ORIENT }} & =\int \frac{\rho(R)}{2}\left(D_{t} R\right)^{2} d R \\
& =\int \frac{\rho(R)}{2}[(\mathcal{W}+\Omega) R]^{2} d R \\
& =\int \frac{\rho(R)}{2}<\mathcal{W}+\Omega,[\tilde{R},[\tilde{R}, \mathcal{W}+\Omega]]>d R \\
& =-\frac{1}{2}<\mathcal{W}+\Omega,\left[\int-\rho(R)(a d \tilde{R})^{2} d R\right](\mathcal{W}+\Omega)> \\
& =-\frac{1}{2}<\mathcal{W}+\Omega, I(t)(\mathcal{W}+\Omega)>,
\end{aligned}
$$


where $\tilde{R} \equiv R^{i} L_{i} \in S O(3)$ and $I(t)=g(t) I_{0} g^{-1}(t)$ and we omitted the explicit expression for the time dependence of the variables. This orientation part also can be written in a compact form as

$$
\mathcal{L}_{\text {ORIENT }}=-\frac{1}{2}<\tilde{S}(t), \mathcal{W}(t)+\Omega(t)>
$$

with the definition $\tilde{S}(t) \equiv g(t) \tilde{S}_{0}(t) g^{-1}(t)=S^{i}(t) L_{i}$. For the local $S O(3)$ transformation the variables transform as;

$$
\begin{aligned}
r(t) & =O(t) R(t)=O(t) L^{-1}(t) L(t) g(t) R_{0} \\
R^{\prime}(t) & =L(t) R(t) \\
O^{\prime}(t) & =O(t) L^{-1}(t) \\
g^{\prime}(t) & =L(t) g(t) \\
\Omega^{\prime}(t) & =L(t) \Omega(t) L^{-1}+L(t) \dot{L}^{-1}(t) \\
\mathcal{W}^{\prime}(t) & =L(t) \mathcal{W}(t) L^{-1}+\dot{L}(t) L^{-1}(t) .
\end{aligned}
$$

We see that $\mathcal{W}(t)+\Omega(t)$ now transforms covariantly which assures that local $S O(3)$ symmetry of the system. It should be noted that for the covariant description we introduce other variables $g(t) \in S O(3)$. Now the Lagrangian for the isotropic rotator reads as

$$
\mathcal{L}_{\text {ROTATOR }}=\frac{1}{2} m \dot{x}^{2}-\frac{1}{2}<\mathcal{W}(t)+\Omega(t), I(t)(\mathcal{W}(t)+\Omega(t))>.
$$

We note that the Lagrangian (6) comes as a specific case;

$$
L(t)=g^{-1}(t) \Rightarrow \mathcal{W}^{\prime}(t)+\Omega^{\prime}(t)=(O(t) g(t))^{-1} \frac{d}{d t}(O(t) g(t)) \equiv s^{-1}(t) \dot{s}(t)
$$

The system in [6] is obtained through the above gauge fixing and with a choice of $S_{0}(t)$ in a specific directionf Further, the meaning of the $U(1)$ symmetry mentioned there become clear with the view that it is the residual subsymmetry of this local internal symmetry $S O(3)$. For another specific case, we take the following 'gauge fixing'.

$$
L(t)=O(t) \Rightarrow \mathcal{W}^{\prime}(t)+\Omega^{\prime}(t)=\frac{d}{d t}(O(t) g(t))(O(t) g(t))^{-1} \equiv \dot{s}(t) s^{-1}(t) .
$$

This corresponds to the description of the system with respect to the non-rotating frame.

\footnotetext{
${ }^{4}$ This is possible because the variation of this $I_{0} s^{-1}(t) \dot{s}(t)$ part just gives extra numerical factor that can be absorbed in the scale of that specific direction.
} 
Now we are in a position to consider the relativistic spinning particle. For the relativistic description, we replace the variables with the relativistic ones. Further, we do without those quantities $\left(I_{0}, R_{0}, I(t), R(t), \iota(t), r(t)\right)$ which designate the extendedness of the rotator since we now want to describe a point particle.

$$
\begin{gathered}
t \rightarrow \tau, O(t) \rightarrow \Lambda(\tau), g(t) \rightarrow \bar{\Lambda}(\tau), \\
\frac{1}{2} m \dot{x}^{2} \rightarrow p \dot{x}, \\
<\tilde{S}(t), \mathcal{W}+\Omega>\rightarrow<\tilde{S}(t), \Lambda^{-1} \dot{\Lambda}+\dot{\bar{\Lambda}} \bar{\Lambda}^{-1}>,
\end{gathered}
$$

where $\tau$ is the invariant time parameter and changes the meaning of dot, and $\Lambda, \bar{\Lambda} \in$ $S O(3,1)$. Now, with the basis $\sigma_{\mu \nu}$ of $s o(3,1), \tilde{S}=S^{\mu \nu} \sigma_{\mu \nu} \in S O(3,1)$ and it concerns the 'internal' angular momentum. We use the first order formalism, accordingly the following constraints should be taken into account.

$$
p^{2}+m^{2}=0, p=\Lambda \bar{\Lambda} m
$$

Here, particle mass $m$ is defined in the 'body-fixed frame that the concept 'non-rotating frame' is changed because four dimensional rotation $\Lambda \bar{\Lambda} \in S O(3,1)$ includes boosting, which makes the 'non-rotating frame' depart from the 'center of mass'. In this relativistic case, we consider the 'non-rotating frame' coincident with the 'observer-rest frame'. To define the spin that is intrinsic to the particle, we fix the gauge so that $\tilde{S}$ is fixed in that chosen rotating frame, like mass $m$ is the fixed momentum in the 'body-fixed frame'.

With the variables described with respect to this $\tilde{S}$ fixed frame, the Lagrangian becomes

$$
\mathcal{L}=p \cdot \dot{x}-\frac{1}{2}<\tilde{\lambda}, \Lambda^{-1} \dot{\Lambda}+\dot{\bar{\Lambda}} \bar{\Lambda}^{-1}>-N\left(p^{2}+m^{2}\right)-M \cdot \tilde{\lambda}\left(\Lambda^{-1} p-\bar{\Lambda} m\right),
$$

where the fixed $\tilde{S}$ is denoted as $\tilde{\lambda}$ and those constraints (18) are (global) Lorentz invariantly put into the Lagrangian with the Lagrangian multipliers $N$ and $M_{\mu}$. The $\tilde{\lambda}$ in the last constraint term accounts for the vanishing of the term for the spinless particle. Written in components, this Lagrangian leads to

$$
\begin{aligned}
\mathcal{L}= & p^{\mu} \dot{x}_{\mu}-\frac{1}{2} \lambda^{\mu \nu} \Lambda^{\sigma}{ }_{\mu} \dot{\Lambda}_{\sigma \nu}-\frac{1}{2} \lambda^{\mu \nu} \dot{\bar{\Lambda}}_{\mu \sigma} \bar{\Lambda}_{\nu}{ }^{\sigma} \\
& -N\left(p^{2}+m^{2}\right)-M_{\mu} \lambda^{\mu \nu}\left(p^{\sigma} \Lambda_{\sigma \nu}-\bar{\Lambda}_{\nu 0} m\right) .
\end{aligned}
$$

\footnotetext{
${ }^{5}$ Since the point particle is bodiless this name is a little absurd, but we still use that name avoiding perfusion of terminology.
} 
It is to be noticed that only $\bar{\Lambda}_{\nu 0}$ components of $\bar{\Lambda}$ couple with other variables through the constraint terms; the components $\bar{\Lambda}_{\nu i}$ can be completely decoupled away. This leaves the following Lagrangian.

$$
\mathcal{L}=p^{\mu} \dot{x}_{\mu}-\frac{1}{2} \lambda^{\mu \nu} \Lambda^{\gamma}{ }_{\mu} \dot{\Lambda}_{\gamma \nu}-\frac{1}{2} \lambda^{\mu \nu} t_{\mu} \dot{t}_{\nu}-N\left(p^{2}+m^{2}\right)-M_{\mu} \lambda^{\mu \nu}\left(p^{\sigma} \Lambda_{\sigma \nu}+m t_{\nu}\right),
$$

where $\bar{\Lambda}_{\nu}{ }^{0}$ is denoted as $t_{\nu}$. This is exactly the same system as the classical model recently proposed in $[7,9$.

We conclude this letter with some remarks. In three dimension, we introduced two kinds of variables: $x^{i}(t)$ denote the position whereas $r^{i}(t)(O(t))$ specify the orientation. For the covariant description under the local internal $S O(3)$, we introduce other variables $g(t)$. Those variables are corresponding to the observer-rest inertial frame, the non-rotating frame and the general rotating frame respectively. The latter two frames are internal in the sense that they are pertaining to the rotator. However, relativistic extension shuffles the observer-rest frame and the non-rotating frame because of the boosting. We put the relation between these two frames through the second constraint in (18). This constraint does the role of bridge to connect those two frame. Indeed, it corresponds to the constraint $p \cdot \psi+m \psi^{*}=0$ of the pseudo-classical formulation and generates 'quasi-supersymmetry' [7, 9].

The equations of motion can be easily derived by the variational principle;

$$
\begin{aligned}
\delta x^{\mu} & \Rightarrow \frac{d}{d \tau} p^{\mu}=0 \\
\delta \Lambda & \Rightarrow \frac{d}{d \tau}\left(x^{\mu} p^{\nu}-x^{\nu} p^{\mu}+\Lambda^{\mu}{ }_{\rho} \Lambda^{\nu}{ }_{\sigma} \lambda^{\rho \sigma}\right)=0 .
\end{aligned}
$$

With the spin component $\Sigma^{\mu \nu}=\Lambda^{\mu}{ }_{\rho} \Lambda_{\sigma}^{\nu} \lambda^{\rho \sigma}$, we can see these are the right equations of motion for the relativistic spinning particle. It can also be shown that the Dirac algebra for the momentum $p^{\mu}$ and the total angular momentum $J^{\mu \nu}=x^{\mu} p^{\nu}-x^{\nu} p^{\mu}+$ $\Sigma^{\mu \nu}$ is isomorphic to the Poincaré algebra [7] and the system gives the same spin factor as the one given by the pseudo-classical model [9].

In this letter, we showed that the classical model for the relativistic spinning particle can be obtained through the relativistic extension of the isotropic rotator. We hope this derivation to be helpful for the intuitive understanding of the 'spin'. 


\section{References}

[1] E. Schrödinger and Sitzunber, Preuss. Akad. Wiss. Phys.-Math. Kl.24 (1930) 418.

[2] A. Hanson, T. Regge and C. Teitelboim, Accademia Nazionale dei Lincei (Roma, 1976).

[3] F.A. Berezin and M.S. Marinov Ann.Phys.104(1977)336; L. Brink, P. Di Vecchia and P. Howe Nucl. Phys. B118(1977)76.

[4] A. O. Barut and Zanghi, Phys. Rev. Lett.52(1984)2009.

[5] A. Barducci, R. Caslbuoni, D. Dominici and R. Gatto, Phys. Lett. B 187 (1987)135; C. Batlle, J. Gomis and J. Roca, Phys. Rev. D40(1989)1950.

[6] A.P. Balachandran, G. Marmo, B.-S. Skagerstam and A. Stern, Gauge Symmetries and Fiber Bundles, (Springer-Verlag, Berlin, 1983).

[7] J.-H. Cho, S.J. Hyun and J.-K. Kim, "A Covariant Formulation of Classical Spinning Particle", hep-th/9302012 (To appear in Mod. Phys. Lett. A).

[8] A. M. Polyakov, Les Houches, Session XLIX,(ed. E. Brézin and J. ZinnJustin,1988); J. Grundberg, T. H. Hansson, A. Karlhede and U. Lindström, Phys. Lett. B218(1989) 321; J. Grundberg, T. H. Hansson, and A. Karlhede, Nucl. Phys. B347(1990)420.

[9] J.-H. Cho, S.J. Hyun and H.-j. Lee, "Polyakov's Spin Factor for a Classical Spinning Particle via BRST Invariant Path Integral”, hep-th/9403103 (To appear in Phys. Lett. B).

[10] D. H. Sattinger and O. L. Weaver, Lie Groups and Algebras with Applications to Physics, Geometry and Mechanic, (Springer-Verlag, New York, 1986).

[11] A. Alekseev and S. Shatashivili, Nucl. Phys. B323(1989)719 